Pure and Applied Mathematics Quarterly

Volume 4, Number 2

(Special Issue: In honor of

Fedor Bogomolov, Part 1 of 2)

$203-236,2008$

\title{
Is There a Topological Bogomolov-Miyaoka-Yau Inequality?
}

\author{
János Kollár
}

Let $S$ be a smooth, complex, projective, minimal surface of general type. The Bogomolov-Miyaoka-Yau inequality states that $c_{1}(S)^{2} \leq 3 c_{2}(S)$ [Bog78, Rei78, Miy77, Yau77]. In this note I want to address the following question:

Is there a topological analog of the Bogomolov-Miyaoka-Yau inequality?

The 11/8-conjecture [Mat82, Fur01] can be viewed as such, but in Section 1 I write down another possible variant. Section 2 explores its relationship with the Montgomery-Yang problem on differentiable circle actions on $S^{5}$ and Section 3 examines its connection with the $\mathrm{H}$-cobordism group of 3-manifolds. Related examples and questions on algebraic surfaces are discussed in Section 4. The last section studies the remarkable series of hypersurfaces

$$
\left(x_{1}^{a_{1}} x_{2}+x_{2}^{a_{2}} x_{3}+\cdots+x_{n-1}^{a_{n-1}} x_{n}+x_{n}^{a_{n}} x_{1}=0\right)
$$

in weighted projective spaces.

The aim of this note is two fold. On the one hand, I would like to call attention to several questions about algebraic surfaces with quotient singularities that have interesting connections with the topology of 3 - or 5-manifolds. The study of algebraic orbifolds led to many interesting examples in topology and differential geometry (see, for instance, [Bri66, OW75] or the recent papers [BGK05, Kol05]), but there should be many more connections.

Received February 24, 2006. 
On the other hand, more speculatively, I hope that methods developed around the Bogomolov-Miyaoka-Yau inequality can be adapted to the topological setting, leading to progress on the questions mentioned in Sections 2 and 3.

\section{The orbifold Bogomolov-Miyaoka-Yau inequality}

The Bogomolov-Miyaoka-Yau inequality can be generalized to orbifolds. These are normal projective surfaces whose singularities are locally analytically isomorphic to quotient singularities $\mathbb{C}^{2} / G$ where $G \subset G L(2, \mathbb{C})$ is a finite group whose action is fixed point free outside the origin. Such a surface has finitely many singular points, and locally at each of them $S$ is topologically the cone over a 3 -manifold $S^{3} / G$ where $G \subset U(2, \mathbb{C})$ is a subgroup acting without fixed points. This 3 -manifold is called the link of $s \in S$ and it is denoted by $L_{s}$. (The version where a surface is allowed to have orbifold structure in codimension one is also interesting [BGK05, Kol05], but it will not be considered here.)

An easy way to get such an orbifold is to take the quotient of a smooth projective surface $X$ by a finite group $G$ acting on $X$ with only isolated fixed points. The most interesting examples are, however, those that do not arise as a global quotient. For a complex surface, $c_{2}(S)$ is the same as the topological Euler characteristic. Following Thurston, we introduce the orbifold Euler characteristic

$$
e_{\text {orb }}(S):=e(S)-\sum_{s \in \operatorname{Sing} S}\left(1-\frac{1}{\left|\pi_{1}\left(L_{s}\right)\right|}\right) .
$$

The orbifold version of the Bogomolov-Miyaoka-Yau inequality is the following, developed in the series of papers [Sak80, Miy84, KNS89, Meg92].

Theorem 1. Let $S$ be a normal projective surface with quotient singularities such that $-c_{1}(S)$ is ample (or at least nef). Then

$$
c_{1}(S)^{2} \leq 3 e_{\text {orb }}(S)
$$

Since $-c_{1}(S)$ is nef, $c_{1}(S)^{2} \geq 0$, thus we also get the following weaker inequality, which also holds when $c_{1}(S)$ is nef by [KM99]

$$
0 \leq e_{\text {orb }}(S)
$$

While (1.2) gives nothing interesting for smooth surfaces, it has very interesting consequences for singular surfaces. 
Let $S$ be a normal projective surface with quotient singularities whose second Betti number is 1 . Then either $\pm c_{1}(S)$ is ample or $c_{1}(S)=0$. Thus (1.2) applies and so $0 \leq e_{\text {orb }}(S)$. It is easy to see (by looking at the Albanese map) that $b_{1}(S)=0$, thus $e(S)=3$ and so $S$ is a rational homology $\mathbb{C P}^{2}$. Thus we get to following expanded version of (1.2):

$$
\sum_{s \in \operatorname{Sing} S}\left(1-\frac{1}{\left|\pi_{1}\left(L_{s}\right)\right|}\right) \leq 3 .
$$

In particular, $S$ has at most 6 singular points.

Let us now make the bold (or foolish) guess that the inequality (1.3) is topological in nature. It may be more convenient to formulate the conjecture for smooth, compact 4-manifolds $M$ whose boundary components are spherical, that is, their universal cover is $S^{3}$. One can then attach cones to each boundary component to get a 4-dimensional orbifold $S$. We are mainly interested in the cases when $S$ is a homology $\mathbb{C P}^{2}$. Correspondingly, $H_{1}(M, \mathbb{Z})=0$ and $H_{2}(M, \mathbb{Z}) \cong \mathbb{Z}$.

Conjecture 2 (Smooth Bogomolov-Miyaoka-Yau inequality). Let $M^{4}$ be a smooth, compact 4-manifold with spherical boundary components $\partial M^{4}=\cup_{i} L_{i}$. Assume that $H_{1}(M, \mathbb{Z})=0$ and $H_{2}(M, \mathbb{Z}) \cong \mathbb{Z}$. Then

$$
\sum_{i}\left(1-\frac{1}{\left|\pi_{1}\left(L_{i}\right)\right|}\right)<3
$$

In particular, $M$ has at most 5 boundary components.

3 (The origins of the conjecture). While, to the best of my knowledge, the above conjecture is new, various forms of it have been implicit in the works of several authors.

Controlling the singularities of a variety by global invariants has been one of the aims of the papers [Miy84, Meg92, KM99]. It is quite natural to go from an algebraic formulation to a purely topological one as above.

On the topological side, the Montgomery-Yang problem on circle actions on 5 -manifold was explicitly studied as a problem on 4-dimensional orbifolds in [FS87]. As we discuss in Section 2, the relationship with (2) is close but the two problems are not identical.

The history of the topological work on H-cobordisms of Seifert fibered 3manifolds is reviewed in [Sav02]. The same topic appears in singularity theory 
as the study of smoothings of quasihomogeneous surface singularities. The link of such a singularity is a Seifert fibered 3-manifold, and each smoothing exhibits the link as the boundary of the Milnor fiber. In this setting, (2) is closely related to [Wah94, 3.10]. His results imply (2) for smoothings of negative weight.

4 (Comments and possible generalizations). 1. In the algebraic case, if equality holds in (1.3) then $c_{1}(S)=0$ and $S$ is the quotient of an Abelian or a K3 surface by a cyclic group. In particular, $H_{1}(S \backslash \operatorname{Sing} S, \mathbb{Z}) \neq 0$. This is why the $\leq$ in (1.3) was changed to a strict inequality in (2.1). An algebraic rational homology $\mathbb{C P}^{2}$ can never have 6 singular points and I don't know any examples with 5 singular points. There are, however, many examples with 4 singular points (31).

2. Unlike in the algebraic case, the restriction $H_{1}(M, \mathbb{Z})=0$ is not a consequence of $H_{2}(M, \mathbb{Z})=\mathbb{Z}$. Indeed, attaching a 1-handle to $M$ increases $H_{1}(M, \mathbb{Z})$ while leaving $H_{2}(M, \mathbb{Z})$ unchanged.

3. The assumption $H_{1}(M, \mathbb{Z})=0$ is somewhat arbitrarily chosen. One could require instead $\pi_{1}(M)=1$. The variant with $H_{1}(M, \mathbb{Z})=0$ is the relevant condition for integral $\mathrm{H}$-cobordism questions and $\pi_{1}(M)=1$ connects directly with the Montgomery-Yang problem on circle actions.

4. In the algebraic case the inequality (2.1) holds even if we only assume that $H_{1}(M, \mathbb{Q})=0$, but in the topological case this is not enough. There are lens spaces which bound a rational homology ball, and taking connected sum of these with $\mathbb{C P}^{2}$ gives examples with an arbitrary number of boundary components.

The simplest algebraic example is the following. Let $C \subset \mathbb{C P}^{2}$ be a smooth conic and $C \subset N$ a regular neighborhood with boundary $L$. Since the normal bundle of $C$ in $\mathbb{C P}^{2}$ has degree 4 , we see that $L$ is a $\mathbb{Z} / 4$-quotient of $S^{3}$. Set $M:=\mathbb{C P}^{2} \backslash \operatorname{Int} N . \quad M$ is a rational homology ball with $\pi_{1}(M)=\mathbb{Z} / 2$ which bounds $L$.

There are many such examples, see, for instance, [CH81].

To get more algebraic ones, let $u, v$ be relatively prime natural numbers. Then the complement of a regular neighborhood of the curve

$$
C:=\left(x^{u+v}-y z=0\right) \subset \mathbb{P}^{2}(1, u, v) .
$$

is a rational homology ball $M$ with $\pi_{1}(M)=\mathbb{Z} /(u+v)$ which bounds a lens space $L$ with $\pi_{1}(L)=\mathbb{Z} /(u+v)^{2}$. 
5. By [FS85, p.360], the Poincaré sphere is not $\mathrm{H}$-cobordant to 0, thus one can not use connected sums to get counter examples to (2).

6. It is possible that Conjecture 2 can be generalized to arbitrary $H_{2}(M, \mathbb{Z})$. In this case, (2.1), should be replaced by

$$
\sum_{i}\left(1-\frac{1}{\left|\pi_{1}\left(L_{i}\right)\right|}\right) \leq 2+\operatorname{dim} H_{2}(M, \mathbb{Q})
$$

This may hold if $\pi_{1}(M)=1$ but - as pointed out to me by P. Hacking $H_{1}(M, \mathbb{Z})=0$ is not sufficient, not even for algebraic surfaces. The surfaces in question arise as the minimal compactifications of Seifert $\mathbb{C}^{*}$-bundles over $\mathbb{P}^{1}$. These were studied by [Dol75, Pin77, Dem88, FZ03].

One of the simplest examples is the following.

Example 5. Rational surfaces $S_{m}$ with $H_{1}\left(S_{m}, \mathbb{Z}\right)=0, H_{2}\left(S_{m}, \mathbb{Z}\right) \cong \mathbb{Z}^{2}$ and with $2 m$ quotient singularities.

Let us start with any minimal ruled surface $f: S \rightarrow \mathbb{P}^{1}$. Pick points $p_{i} \in F_{i} \subset S$ where the $F_{i}$ are different fibers of $f$ and natural numbers $r_{i}$. Blow up $\left(2+r_{i}\right)$ times each $p_{i} \in F_{i}$ and in each fiber contract all but the last $(-1)$-curve. We get a singular ruled surface

$$
g=g\left(r_{1}, \ldots, r_{m}\right): S\left(r_{1}, \ldots, r_{m}\right) \rightarrow \mathbb{P}^{1} \quad \text { with } \quad H_{2}\left(S\left(r_{1}, \ldots, r_{m}\right), \mathbb{Q}\right) \cong \mathbb{Q}^{2} .
$$

The fiber over $f\left(p_{i}\right)$ is a smooth rational curve with multiplicity $r_{i}$. Moreover, $S\left(r_{1}, \ldots, r_{m}\right)$ has 2 singular points along these fibers. They are cyclic quotients of the form $\mathbb{C}^{2} / \frac{1}{r_{i}}(1,1)$ and $\mathbb{C}^{2} / \frac{1}{r_{i}}(1,-1)$.

It is easy to see that the fundamental group of the smooth part is given by generators and relations as:

$$
\pi_{1}\left(S\left(r_{1}, \ldots, r_{m}\right)^{0}\right) \cong\left\langle a_{1}, \ldots, a_{m}: a_{1}^{r_{1}}=\cdots=a_{m}^{r_{m}}=a_{1} \cdots a_{m}=1\right\rangle .
$$

If the $r_{i}$ are pairwise relatively prime then $H_{2}\left(S\left(r_{1}, \ldots, r_{m}\right), \mathbb{Z}\right) \cong \mathbb{Z}^{2}$ but the fundamental group is never trivial for $m \geq 3$.

It is worth noting that (2) completely fails if $M$ is only a topological manifold. The number of boundary components can be arbitrary, see (25). 


\section{The Montgomery-Yang Problem}

Fixed point free differentiable circle actions on $S^{7}$ with finitely many nonfree orbits are classified in [MY72], see also [Pet75]. Such actions are frequently called pseudo-free. The main idea of their classification is the following.

We work on $S^{7}$ and on the orbifold quotient $X:=S^{7} / S^{1}$. Let $O_{i} \subset S^{7}$ be a nonfree orbit and $x_{i} \in X$ the corresponding orbifold point. $O_{i}$ bounds a disk in $S^{7}$ whose image is a 2 -sphere $S_{i} \subset X$ containing $x_{i}$. Since $\operatorname{dim} X>4$, we can arrange these $S_{i}$ to be disjoint. The classification now has 2 parts.

i) Describe the circle action over a neighborhood of each $S_{i}$. These turn out to be diffeomorphic to linear circle actions on $S^{3} \times D^{4}$.

ii) Describe how these local models glue together.

By contrast, if we work on $S^{5}$, then $\operatorname{dim} S^{5} / S^{1}=4$, thus the different $S_{i}$ do intersect, and we can not separate the local models from each other. (Note that if a manifold $M$ admits a fixed point free differentiable circle action, then $e(M)=0$, thus this theory is most interesting for odd dimensional manifolds.)

Montgomery and Yang found that it is difficult to construct examples of differentiable circle actions on $S^{5}$ with many nonfree orbits. This led to the following:

Conjecture 6 (Montgomery-Yang problem). [MY72, p.41] Let $S^{1} \times S^{5} \rightarrow S^{5}$ be a differentiable circle action with only finitely many nonfree orbits. Then there are at most 3 nonfree orbits.

(Note that the proposed partial solution in [FS87] is incorrect, see the review, MR0874031.)

There are many different actions with 3 exceptional orbits. The simplest ones are linear actions but there are many nonlinear examples too.

Example 7. Let $S^{5}=\left(|x|^{2}+|y|^{2}+|z|^{2}=1\right) \subset \mathbb{C}^{3}$ be the unit sphere. Let $a, b, c \geq 2$ be pairwise relatively prime natural numbers. Then $S^{1} \times S^{5} \rightarrow S^{5}$, given by

$$
(\lambda, x, y, z) \mapsto\left(\lambda^{b c} x, \lambda^{c a} y, \lambda^{a b} z\right)
$$

is a differentiable circle action with 3 nonfree orbits on the 3 coordinate axes.

Note that in this case the quotient $S^{5} / S^{1}$ can be thought of as the (complex) weighted projective plane $\mathbb{P}^{2}(a, b, c)$. 
In general, one can try to classify circle actions on 5-manifolds $L$ in terms of the 4-dimensional quotient orbifold $L / S^{1}$.

Let $L$ be a 5 -manifold and $S^{1} \times L \rightarrow L$ a differentiable circle action. Assume that there are finitely many $S^{1}$-orbits $O_{1}, \ldots, O_{m}$ such that the action is free on $L \backslash \cup_{i} O_{i}$. For each $O_{i} \subset L$ take an $S^{1}$-invariant tubular neighborhood $O_{i} \subset T_{i} \subset$ $L$. Then $L^{0}:=L \backslash \cup_{i} T_{i}$ is a compact 5 -manifold with boundary and with a free circle action. Thus $M:=L^{0} / S^{1}$ is a compact 4 -manifold with boundary, where the boundary components are lens spaces.

The 4-manifold $M$ uniquely determines $L$ and the $S^{1}$-action up to diffeomorphism in many cases. See [MY72, FS85] for the pseudo-free case and [Kol05] in general. (While [Kol05] considers only the algebraic case, the result is valid in general using the methods of [Kol06].) One of the simplest cases is pseudo-free circle actions on 5-dimensional rational homology spheres.

Theorem 8. There is a one-to-one correspondence between:

(1) Pseudo-free differentiable circle actions on 5-dimensional rational homology spheres $L$ with $H_{1}(L, \mathbb{Z})=0$.

(2) Smooth, compact 4-manifolds $M$ with boundary such that

(a) $\partial M=\cup_{i} L_{i}$ is a disjoint union of lens spaces $L_{i}=S^{3} / \mathbb{Z}_{m_{i}}$,

(b) the $m_{i}$ are relatively prime to each other,

(c) $H_{1}(M, \mathbb{Z})=0$ and $H_{2}(M, \mathbb{Z}) \cong \mathbb{Z}$.

Furthermore, $L$ is diffeomorphic to $S^{5}$ iff $\pi_{1}(M)=1$.

The smooth Bogomolov-Miyaoka-Yau-type conjecture 2 would give the following for circle actions:

Conjecture 9. Let $L$ be a 5-dimensional rational homology sphere with $H_{1}(L, \mathbb{Z})=$ 0 admitting a pseudo-free differentiable circle action. Let $O_{1}, \ldots, O_{k}$ be the nonfree orbits with stabilizers $\mathbb{Z} / m_{1}, \ldots, \mathbb{Z} / m_{k}$. Then

$$
\sum_{i}\left(1-\frac{1}{m_{i}}\right) \leq 3 \text {. }
$$

In fact, using [Kol06], one can generalize this to fixed point free actions which are not pseudo-free. Let us say that an orbit $O \subset L$ of an $S^{1}$-action is exceptional if the order of its stabilizer $|\operatorname{stab}(O)|$ is bigger than the least common multiple 
of the orders of the stabilizers of nearby orbits, that is $\operatorname{lcm}\left\{\left|\operatorname{stab}\left(O_{p}\right)\right|: p \notin O\right\}$. It is easy to see that the quotient

$$
m(O):=|\operatorname{stab}(O)| / \operatorname{lcm}\left\{\left|\operatorname{stab}\left(O_{p}\right)\right|: p \notin O\right\}
$$

is an integer which is also the order of the local fundamental group of $L / S^{1}$ at the image of $O$ [Kol06, Prop.15]. Thus (2) would imply the following generalization of (9):

Conjecture 10. Let $L$ be a 5-dimensional rational homology sphere with $H_{1}(L, \mathbb{Z})=$ 0 admitting a fixed point free differentiable circle action. Let $O_{1}, \ldots, O_{k}$ be the exceptional orbits. Then

$$
\sum_{i}\left(1-\frac{1}{m\left(O_{i}\right)}\right) \leq 3
$$

11. A positive answer to Conjecture 2 would come close to settling the MontgomeryYang problem. Indeed, first we enumerate all sequences of pairwise relatively prime natural numbers $m_{1}, \ldots, m_{k}$ such that

$$
\sum_{i=1}^{k}\left(1-\frac{1}{m_{i}}\right) \leq 3 .
$$

The list turns out to be very short and we get one of the following cases:

(1) $k \leq 3$, as required by the Montgomery-Yang problem,

(2) $(2,3,5, n)$ for any $(n, 30)=1$,

(3) $(2,3,7, n)$ for $n \in\{11,13,17,19,23,25,29,31,37,41\}$, or

(4) $(2,3,11,13)$.

So, right away we get that there are at most 4 nonfree orbits (assuming Conjecture $2)$.

I do not know any 4 -manifolds $M$ with $H_{1}(M, \mathbb{Z})=0$ corresponding to the cases (2-4) above but I can not exclude these even in the algebraic case. (See, however, (31) for some examples where one of the singularities is not a cyclic quotient.)

Even if such a manifold exists, it leads to a counterexample to the MontgomeryYang problem only if it is simply connected.

The methods to prove (8) lead to a complete characterization of compact, simply connected 5-manifolds which admit a fixed point free differentiable circle action. Before we state the result, we need to define an invariant for 5-manifolds. 
Definition 12. For any manifold $L$, write its second homology as a direct sum of cyclic groups of prime power order

$$
H_{2}(L, \mathbb{Z})=\mathbb{Z}^{k}+\sum_{p, i}\left(\mathbb{Z} / p^{i}\right)^{c\left(p^{i}\right)} \quad \text { for some } k=k(L), c\left(p^{i}\right)=c\left(p^{i}, L\right) .
$$

The numbers $k, c\left(p^{i}\right)$ are uniquely determined by $H_{2}(L, \mathbb{Z})$. One can choose the decomposition such that the second Stiefel-Whitney class map

$$
w_{2}: H_{2}(L, \mathbb{Z}) \rightarrow \mathbb{Z} / 2
$$

is zero on all but one summand $\mathbb{Z} / 2^{n}$. This value $n$ is unique and it is denoted by $i(L)$ [Bar65]. Alternatively, $i(L)$ is the smallest $n$ such that there is an $\alpha \in$ $H_{2}(L, \mathbb{Z})$ such that $w_{2}(\alpha) \neq 0$ and $\alpha$ has order $2^{n}$.

By [Sma62, Bar65], a compact, simply connected 5-manifolds is determined by the invariants $k(L), c\left(p^{i}, L\right)$ and $i(L)$.

Theorem 13. [Kol06, Thm.3] Let L be a compact, simply connected 5-manifold with invariants $k(L), c\left(p^{i}, L\right), i(L)$ as in (12.1). Then $L$ admits a fixed point free differentiable circle action iff the following conditions hold:

(1) for every prime $p$, there are at most $k+1$ nonzero $c\left(p^{i}\right)$,

(2) $i(L) \in\{0,1, \infty\}$, and

(3) if $i(L)=\infty$ then there are at most $k$ nonzero $c\left(2^{i}\right)$.

14 (Comments). 1. The above result gives a complete characterization of those manifolds that admit a fixed point free circle action, but it does not describe all possible circle actions. For any $L$ satisfying the above assumptions, infinitely many topologically distinct circle actions are constructed in [Kol06] and a complete classification seems unlikely.

2. The circle actions constructed in [Kol06] have 2-parameter families of nonfree orbits. Pseudo free actions exist only if $H_{2}(L, \mathbb{Z})$ is torsion free (cf. [Kol06, Prop.28]).

\section{H-COBORDISM OF 3-MANIFOLDS}

Definition 15. Let $\Sigma$ be an integral homology sphere, that is $H_{*}(\Sigma, \mathbb{Z})=$ $H_{*}\left(S^{3}, \mathbb{Z}\right) . \Sigma$ is $H$-cobordant to zero if there is a smooth 4-dimensional homology cell $W$ with boundary $\partial W=\Sigma$. Similarly, one can study rational homology spheres which are rationally $\mathrm{H}$-cobordant to zero. 
It is an interesting and difficult question to decide which integral/rational homology spheres are H-cobordant to zero; see [Sav02, Chap.7] for a survey.

The case when $\Sigma$ is Seifert fibered is very much connected with the BogomolovMiyaoka-Yau inequality.

Definition 16 (Seifert fiber spaces).

A Seifert fibered 3-manifold is a proper morphism of a 3-manifold to a surface $f: M \rightarrow S$ such that every point $s \in S$ has a neighborhood $s \in D_{s} \subset S$ such that the pair $f^{-1}\left(D_{s}\right) \rightarrow D_{s}$ is fiber preserving homeomorphic to one of the normal forms $f_{c, d}$ defined as follows.

Let $S^{1}(z), D^{2}(z) \subset \mathbb{C}$ denote the unit circle (resp. open unit disk) where $(z)$ indicates the name of the coordinate. For a pair of integers $c, d$ satisfying $0 \leq$ $c<d$ and $(c, d)=1$, define

$$
f_{c, d}: S^{1}(u) \times D^{2}(z) \rightarrow D^{2} \quad \text { by } \quad f_{c, d}(u, z)=u^{c} z^{d} .
$$

$f_{c, d}$ restricts to a fiber bundle $S^{1} \times\left(D^{2} \backslash\{0\}\right) \rightarrow D^{2} \backslash\{0\}$. The fiber of $f_{c, d}$ over the origin is still $S^{1}$, but $f_{c, d}^{-1}(0)$ has multiplicity $d$.

A Seifert fibered $3-$ manifold $M \rightarrow F$ is determined by the location of the singular fibers $p_{i} \in F$, the above numbers $\left(c_{i}, d_{i}\right)$ at each point $p_{i}$ and by a global invariant, see [Sei32, ST80, Sco83]. For us the following consequence of the classification is more important:

Proposition 17. Given pairwise relatively prime natural numbers $d_{1}, \ldots, d_{k}$, there is a unique integral homology sphere $\Sigma\left(d_{1}, \ldots, d_{k}\right)$ which has a Seifert fibered structure over $S^{2}$ with fiber multiplicities $d_{1}, \ldots, d_{k}$.

18 (Seifert disk bundles). Let $\epsilon$ be a fixed $d$ th root of unity and $\mu_{d} \subset \mathbb{C}$ the group of $d$ th roots of unity. Consider the quotient

$$
D^{2}(x) \times D^{2}(y) / \mu_{d}\left(\epsilon, \epsilon^{c}\right),
$$

where we use this shorthand to denote the quotient of $D^{2}(x) \times D^{2}(y)$ be the $\mu_{d}$-action $(x, y) \mapsto\left(\epsilon x, \epsilon^{c} y\right)$. The second projection map $y \rightarrow y^{d}$ descends to

$$
F_{c, d}: D^{2}(x) \times D^{2}(y) / \mu_{d}\left(\epsilon, \epsilon^{c}\right) \rightarrow D^{2}(y) / \mu_{d}\left(\epsilon^{c}\right) \cong D^{2}\left(y^{d}\right) .
$$

Restricting to $S^{1}(x) \times D^{2}(y)$ we get isomorphisms

$$
S^{1}(x) \times D^{2}(y) / \mu_{d}\left(\epsilon, \epsilon^{c}\right) \cong S^{1}(x) \times D^{2}\left(x^{-c} y\right) / \mu_{d}(\epsilon, 1) \cong S^{1}\left(x^{d}\right) \times D^{2}\left(x^{-c} y\right),
$$


and setting $u=x^{d}, z=x^{-c} y$ the projection map $F_{c, d}$ becomes

$$
(u, v) \mapsto u^{c} z^{d}=\left(x^{d}\right)^{c}\left(x^{-c} y\right)^{d}=y^{d} .
$$

Thus we conclude that $f_{c, d}$ can be extended to the projection map $F_{c, d}$.

We can glue these together and obtain that every Seifert fibered 3-manifold $M \rightarrow F$ can be obtained as the boundary of a Seifert disk bundle $D(M)$. The multiple fibers of multiplicity $m$ correspond to cyclic quotient singularities $\mathbb{C}^{2} / \mu_{d}\left(\epsilon, \epsilon^{c}\right)$ on $D(M)$.

Note that the zero section gives an embedding $F \hookrightarrow D(M)$ and $D(M)$ retracts to $F$. The description of $M$ can also be given in terms of the self intersection of $F \subset D(M)$.

Lemma 19. Let $M \rightarrow F=S^{2}$ be a Seifert fibered 3-manifold with pairwise relatively prime fiber multiplicities $d_{1}, \ldots, d_{k}$ and corresponding Seifert disk bundle $D(M)$. Then

$$
(F \cdot F)= \pm \frac{\left|H_{1}(M, \mathbb{Z})\right|}{d_{1} \cdots d_{k}}
$$

There are several series of examples of Seifert fibered homology spheres which are H-cobordant to zero [AK79, CH81, Ste78, Neu80], but for all of them the number of singular fibers is $\leq 3$. (See also [Ore97].) Let us make the lack of known examples into a formal conjecture:

Conjecture 20. Let $M \rightarrow S^{2}$ be a Seifert fibered rational homology sphere with fiber multiplicities $d_{1}, \ldots, d_{k}$.

(1) If $M$ is rationally $H$-cobordant to zero then $\sum\left(1-\frac{1}{d_{i}}\right)<3$.

(2) If $M=\Sigma\left(d_{1}, \ldots, d_{k}\right)$ and $M$ is $H$-cobordant to zero then $k \leq 3$.

It was observed in [FS87] that this problem is closely related to the existence of 4-manifolds whose boundary components are lens spaces, and hence to Conjecture 2 .

Assume that $\Sigma\left(d_{1}, \ldots, d_{k}\right)$ is the boundary of a homology cell $W$. Above we wrote $\Sigma\left(d_{1}, \ldots, d_{k}\right)$ as the boundary of a Seifert disk bundle $D\left(\Sigma\left(d_{1}, \ldots, d_{k}\right)\right)$. Gluing them together, we get a 4 -dimensional orbifold $S$. The Mayer-Vietoris sequence shows that $H_{*}(S, \mathbb{Z})=H_{*}\left(\mathbb{C P}^{2}, \mathbb{Z}\right)$. Thus $(2)$ implies that we have at most 4 singular points. 
Note, however, that (20.2) is not equivalent to (2). If $S$ is a 4 -dimensional orbifold then usually one can not find an embedded copy of $S^{2}$ passing through all singular points whose regular neighborhood is a Seifert disk bundle.

Example 21 (H-cobordisms of lens spaces). We give 2 algebraic constructions showing that the lens spaces

$$
\begin{aligned}
L_{\left(n^{2}, n c-1\right)} & :=S^{3} /\left(\mathbb{Z} / n^{2}\right)(1, n c-1) \\
& :=\left(|x|^{2}+|y|^{2}=1\right) /(x, y) \mapsto\left(\epsilon x, \epsilon^{n c-1} y\right) \quad \text { where } \quad \epsilon^{n^{2}}=1,
\end{aligned}
$$

are rationally $\mathrm{H}$-cobordant to 0 for any $(c, n)=1$.

The first construction uses deformation of singularities as in [Wah80] or [KSB88, Sec.3]. Under the action

$$
(x, y) \mapsto\left(\epsilon x, \epsilon^{n c-1} y\right) \quad \text { where } \quad \epsilon^{n^{2}}=1,
$$

the subgroup of $n$th roots of unity acts as $(x, y) \mapsto\left(\epsilon^{n} x, \epsilon^{-n} y\right)$, and the quotient is

$$
\left(u v-w^{n}=0\right) \subset \mathbb{C}^{3} \quad \text { where } \quad u=x^{n}, v=y^{n}, w=x y .
$$

The induced quotient action of $\mathbb{Z} / n \cong\left(Z / n^{2}\right) /(\mathbb{Z} / n)$ is given by

$$
(u, v, w) \mapsto\left(\epsilon^{n} u, \epsilon^{-n} v, \epsilon^{n c} w\right)
$$

The Euler characteristic of the Milnor fiber $\left(u v-w^{n}=1\right)$ is $n$, hence the Euler characteristic of the quotient Milnor fiber $\left(u v-w^{n}=1\right) /(\mathbb{Z} / n)$ is 1 . Therefore it is a rational homology ball.

The second construction uses the curve

$$
C:=\left(x y=z^{a+b}\right) \subset \mathbb{P}^{2}(a, b, 1) .
$$

$C$ is smooth, rational and passes through 2 quotient singularities. Thus the bounday $M(a, b)$ of its tubular neighborhood is Seifert fibered over $C$ with 2 multiple fibers, hence it is a lens space. Since $(C \cdot C)=(a+b)^{2} / a b$, we see from (19) that $\left|H_{1}(L, \mathbb{Z})\right|=(a+b)^{2}$.

One needs some explicit computations to decide which lens space. The chart $z \neq 0$ is isomorphic to $\mathbb{C}^{2}$ with coordinates $X:=x z^{-a}, Y:=y z^{-b}$. The affine equation of $C$ is $(X Y=1)$.

$\mathbb{P}^{2}(a, b, 1) \backslash(x=0) \cup C$ is isomorphic to $\mathbb{C}^{*} \times \mathbb{C}$ and one can choose the isomorphism such that in the $X, Y$-coordinates the corresponding coordinate functions 
are

$$
(X Y-1)^{a} X^{-a-b} \text { and } X^{b^{\prime}-a^{\prime}}(X Y-1)^{-b^{\prime}}
$$

where $a a^{\prime}+b b^{\prime}=1$. Correspondingly, $\mathbb{P}^{2}(a, b, 1) \backslash(y=0) \cup C$ is also isomorphic to $\mathbb{C}^{*} \times \mathbb{C}$ and the corresponding coordinate functions are

$$
(X Y-1)^{b} Y^{-a-b} \text { and } Y^{a^{\prime}-b^{\prime}}(X Y-1)^{-a^{\prime}} .
$$

These determine how the two charts are patched together along

$$
\mathbb{C}^{*} \times \mathbb{C}^{*} \cong \mathbb{P}^{2}(a, b, 1) \backslash(x y=0) \cup C
$$

with coordinate functions

$$
X Y(X Y-1)^{-1} \text { and } X^{b} Y^{-a} .
$$

A somewhat messy explicit computation gives that one gets the lens space

$$
M(a, b) \cong L_{(a+b)^{2},\left(a^{\prime}-b^{\prime}\right)(a+b)-1} .
$$

Since $a a^{\prime}+b b^{\prime}=1$, we get that $(a+b) a^{\prime}-\left(a^{\prime}-b^{\prime}\right) b=1$, thus $a+b$ and $a^{\prime}-b^{\prime}$ are relatively prime.

It is easier to see this by putting together the two descriptions as follows. Fix $n$ and $a, c$ such that $a c \equiv 1 \bmod n$. Consider the family of weighted affine hypersurfaces

$$
X(\lambda):=\left(x y-z^{n}+\lambda t=0\right) \subset \mathbb{P}(a, n-a, 1, n) \backslash(t=0) .
$$

For $\lambda=0$ we get

$$
X(0)=\left(x y-z^{n}=0\right) / \frac{1}{n}(a, n-a, 1) \cong\left(x y-z^{n}=0\right) / \frac{1}{n}(1,-1, c) .
$$

For $\lambda \neq 0$ we can eliminate $t$ to get that

$$
X(\lambda) \cong \mathbb{P}(a, n-a, 1) \backslash\left(x y-z^{n}=0\right) .
$$

Thus the quotient Milnor fiber of the first constructions is isomorphic to the complement of the curve $C$ in the second construction.

The following series of [Wah81, 5.9.2] gives an algebraic realization of the rational $\mathrm{H}$-cobordisms constructed in [Neu80, p.132]. See (41) for its projective version. 
Example 22. Let $a, b, c \geq 1$ be integers. Set $S_{a b c}:=\left(x^{a} y+y^{b} z+z^{c} x=0\right) \subset \mathbb{C}^{3}$. $S_{a b c}$ is quasi homogeneous with weights $(b c-c+1, c a-a+1, a b-b+1)$, thus its link is a Seifert fibered 3-manifold $L_{a b c} \rightarrow S^{2}$ with 3 multiple fibers of multiplicities $a b-b+1, b c-c+1, c a-a+1$. The Milnor fiber is

$$
M_{a b c}:=\left(x^{a} y+y^{b} z+z^{c} x=1\right) \subset \mathbb{C}^{3},
$$

and its Euler characteristic is $a b c+1$. Let $\omega$ be a primitive $(a b c+1)$ st root of unity. The cyclic group $\mathbb{Z} /(a b c+1)$ acts freely on $M_{a b c}$ by

$$
(x, y, z) \mapsto\left(\omega x, \omega^{-a} y, \omega^{a b} z\right) .
$$

The quotient $M_{a b c} /(\mathbb{Z} /(a b c+1))$ has Euler characteristic 1 , thus it is a rational homology ball. It bounds the Seifert fibered 3-manifold $L_{a b c} /(\mathbb{Z} /(a b c+1))$ which also has 3 multiple fibers of multiplicities $a b-b+1, b c-c+1, c a-a+1$.

The following remarkable example of [Wah83] shows that there are even examples with 4 singular fibers. Further such examples are in [SSW06].

Example 23 (J. Wahl). A Seifert fibered rational homology sphere with 4 multiple fibers which is rationally $\mathrm{H}$-cobordant to 0 .

The classification of all finite subgroups of $O(n)$ which act freely on the sphere $S^{n-1}$ is the spherical space form problem. See [Wol67, Part III] for a thorough treatment. One such action is obtained as follows (cf. [Wol67, 5.5.6]).

Fix an integer $u \geq 2$ and set

$$
m:=3 u^{2}-3 u+1, n:=9 u, r:=3 u^{2}-6 u+2, a:=3 u-1 .
$$

Let $\zeta$ (resp. $\omega$ ) be a primitive $m$ th (resp. $3 u$ th) root of unity and consider the subgroup $G \subset G L(3, \mathbb{C})$ generated by

$$
A:=(x, y, z) \mapsto\left(\zeta x, \zeta^{r} y, \zeta^{r^{2}} z\right) \quad \text { and } \quad B:=(x, y, z) \mapsto\left(\omega^{-1} z, x, y\right) .
$$

$G$ is nilpotent, its order is $n m$ and it is given by the relations

$$
\left\langle A, B: A^{m}=B^{n}=1, B A B^{-1}=A^{r}\right\rangle .
$$

Consider now the function

$$
f(x, y, z):=x^{a} y+y^{a} z+\omega z^{a} x .
$$

Claim. Notation as above. 
(1) The link of the singularity $(f=0) / G$ is Seifert fibered with 4 singular fibers of multiplicities $(3,3,3, m)$.

(2) The Milnor fiber $(f=1) / G$ is a rational homology ball.

Proof. The singularity $(f=0)$ can be resolved by 1 blow up. The exceptional curve $C$ is smooth of genus $\left(\begin{array}{l}a \\ 2\end{array}\right)$.

Note that $B^{3}=\omega \mathbf{1}$ acts trivially on $C$. The group $G /\left\langle B^{3}\right\rangle$ acts on $C$ with 4 nonfree orbits. These are:

- the orbits of $\left(1: \lambda \omega: \lambda^{2} \omega\right)$ where $\lambda^{3} \omega=1$; these have order 3 stabilizers, and

- the orbit of $(1: 0: 0)$; this has order $m$ stabilizer.

The Hurwitz formula now shows that $C / G \cong \mathbb{P}^{1}$. Moreover $B_{0} \mathbb{C}^{3} / G$ has 4 singular points, three have index 3 and one has index $m$. This shows the first part.

To see the second part, note that by explicit computation, the Milnor number of $f$ is

$$
\operatorname{dim} \mathbb{C}[x, y, z] /(\partial f / \partial x, \partial f / \partial y, \partial f / \partial z)=a^{3} .
$$

Thus the Euler characteristic of the Milnor fiber $(f=1)$ is $1+a^{3}=m n=|G|$. Therefore the Euler characteristic of the quotient Milnor fiber $(f=1) / G$ is 1 , and hence it is a rational homology ball.

24 (Smoothing surface singularities). Let $(0 \in S)$ be a normal surface singularity with link $L$ and let $M$ be the Milnor fiber of a smoothing of $S$. With suitable care, $M$ is a $4-$ manifold whose boundary is $L$. We are especially interested in the case when $M$ is a rational homology ball.

This imposes a very strong restriction on $(0 \in S)$. For instance, it seems to have been known for some time that $(0 \in S)$ is a rational singularity, cf. [SSW06, Sec.2.3]. The latter also shows that (20.1) holds for the link of a normal surface singularity which has a smoothing whose Milnor fiber is a rational homology ball.

For other relationships between the algebraic geometry of a surface singularity and the Seiberg-Witten theory of its link see [NW90, Wah90, NN05, NN04, NN02]. 
Remark 25. By the results of [Fre82], every integral homology sphere bounds a topological homology 4-cell. By the above construction this implies that there are topological integral homology $\mathbb{C P}^{2}$-s with any number of singular points.

\section{Open problems on Algebraic surfaces}

On the algebraic geometry side, all of the questions can be gathered into one central problem:

Problem 26. Classify all integral/rational homology $\mathbb{C P}^{2}$-s with quotient singularities.

I do not expect this to be feasible. The case when the canonical class is anti ample, that is, we are looking at log-Del Pezzo surfaces, received a lot of attention, but there seem to be too many cases for a complete structure theorem; see [Miy01, KM99, Sho00].

The case where the canonical class is numerically trivial should be hard but there is a clear path to follow. If $m K_{S} \sim 0$ then $S$ has a degree $m$ cover which is either an Abelian surface or a K3 surface with Du Val singularities. Thus the problem reduces to the classification of cyclic group actions on Abelian and K3 surfaces. The 7 cases where the Picard number of the K3 surface is maximal are classified in [OZ99].

Very little is known about the case when the canonical class is ample. The recent classification in the smooth case [PY05] is very significant, but it probably says very little about the singular case.

The following examples are worked out in (43).

Example 27. Let $a_{1}, a_{2}, a_{3}, a_{4}$ be natural numbers such that $a_{2} a_{3} a_{4}-a_{3} a_{4}+a_{4}-1$ and $a_{1} a_{2} a_{3} a_{4}-1$ are relatively prime. (This holds in at least $75 \%$ of all cases.) Let $S$ be the surface

$$
S:=S\left(a_{1}, \ldots, a_{4}\right):=\left(x_{1}^{a_{1}} x_{2}+x_{2}^{a_{2}} x_{3}+x_{3}^{a_{3}} x_{4}+x_{4}^{a_{4}} x_{1}=0\right) \subset \mathbb{P}\left(w_{1}, \ldots, w_{4}\right)
$$

where, using subscripts modulo 4,

$$
w_{i}=a_{i+1} a_{i+2} a_{i+3}-a_{i+2} a_{i+3}+a_{i+3}-1 .
$$


Note that $S$ contains the curves $\left(x_{1}=x_{3}=0\right)$ and $\left(x_{2}=x_{4}=0\right)$ and they can be contracted. Thus we obtain a surface $S^{*}=S^{*}\left(a_{1}, a_{2}, a_{3}, a_{4}\right)$. We compute in Section 5 that:

(1) $S^{*}$ is a rational surface with quotient singularities,

(2) $H_{*}\left(S^{*}, \mathbb{Q}\right)=H_{*}\left(\mathbb{C P}^{2}, \mathbb{Q}\right)$,

(3) if $a_{i} \geq 4$ then the canonical class of $S^{*}$ is ample, and

(4) $\left(K_{S^{*}}^{2}\right)$ converges to 1 as $\min \left\{a_{i}\right\} \rightarrow \infty$.

Let us also note that in positive characteristic one can get examples with many quotient singularities.

Example 28 (Characteristic $p$ ). Let $k$ be a field of characteristic $p$. For some $q=p^{m}$, blow up all the $\mathbb{F}_{q}$-points of $\mathbb{P}^{2}$. The birational transforms of the $\mathbb{F}_{q}$-lines become disjoint, smooth, rational curves with self intersection $-q$. They can be contracted to obtain a surface $X_{q}$ defined over $\mathbb{F}_{q}$ with $q^{2}+q+1$ singular points. These are quotient singularities of the type $\mathbb{A}^{2} / \mu_{q}$ where $\mu_{q}$ is the subgroup scheme of $q$ th roots of unity Spec $k\left[t, t^{-1}\right] /\left(t^{q}-1\right)$. (Note that in characteristic $p$ it is the $\mathbb{Z} / q$ quotients that behave very badly (cf. [Art75]) and the $\mu_{q}$-quotients are the correct characteristic $p$ analogs of characteristic 0 quotient singularities.)

$X_{2}$ is a Del Pezzo surface of degree 2, but the canonical class is ample for $q \geq 3$.

Below I list some special cases of Problem 26 which are of interest either as partial steps in the classification or as having immediate topological consequences.

Conjecture 29. [Kol05, 4.17] Let $S$ be a rational homology $\mathbb{C P}^{2}$ with quotient singularities. If $S^{0}:=S \backslash \operatorname{Sing} S$ is simply connected then $S$ is rational.

This is similar in spirit to the result that in dimension 2 , all algebraic $\mathbb{Q}$ homology cells are rational [GP99]. (29) was verified by [Keu05] when the singularities are not very complicated.

Conjecture 30 (Algebraic Montgomery-Yang problem). Let $S$ be a rational homology $\mathbb{C P}^{2}$ with quotient singularities. If $S^{0}:=S \backslash \operatorname{Sing} S$ is simply connected then $S$ has at most 3 singular points.

Example 31. Let $G \subset S L(2, \mathbb{C})$ be subgroup which contains no quasi-reflections such that its image in $\operatorname{PSL}(2, \mathbb{C}$ ) is the icosahedral group $I$ (see [Bri68] for a 
complete list and the corresponding quotient singularities). Let $Z \subset G$ be its center, then $G / Z \cong I$. Extend the $G$-action on $\mathbb{C}^{2}$ to $\mathbb{C P}^{2}$. The center acts trivially on the line at infinity and $\mathbb{C P}^{2} / Z$ is a cone over the rational normal curve of degree $|Z|$. Then $S_{G}:=\mathbb{C P}^{2} / G=\left(\mathbb{C P}^{2} / Z\right) / I$ has 4 quotient singularities, one of type $\mathbb{C}^{2} / G$ at the origin, 3 of types $\mathbb{C}^{2} / \mathbb{Z}_{2}, \mathbb{C}^{2} / \mathbb{Z}_{3}, \mathbb{C}^{2} / \mathbb{Z}_{5}$ at infinity. The fundamental group of $S_{G}^{0}$ is $I$, thus $S_{G}$ is an integral homology $\mathbb{C P}^{2}$.

These examples can also be obtained by starting with a minimal ruled surface and blowing up inside 3 of the fibers.

Problem 32. Classify all integral/rational homology $\mathbb{C P}^{2}$-s with 4 or more quotient singularities.

It is also of considerable interest to study algebraic surfaces that lead to $\mathrm{H}-$ cobordisms of Seifert fibered manifolds. If we want to stay completely algebraic, then we are lead to the following

Problem 33. Classify all pairs $(S, C)$ such that

(1) $S$ is a rational homology $\mathbb{C P}^{2}$,

(2) $C$ is a rational curve, homeomorphic to $S^{2}$.

In this case $S \backslash C$ is a rational homology plane or a $\mathbb{Q}$-acyclic surface. That is a (nonproper) surface $X$ such that $H_{*}(X, \mathbb{Q})=H_{*}\left(\mathbb{C}^{2}, \mathbb{Q}\right)$.

There is a huge body of literature devoted to classifying integral/rational homology planes. See [Fuj82, MT87, tDP89, GS89a, GS89b, FZ94, GP99, DR01a, DR01b, DR04] and the many references there. Nonetheless, most rational homology planes can not be compactified to get a rational homology $\mathbb{C P}^{2}$, thus (33) may be a much easier problem.

Problem 34. Topological smoothings of surfaces with quotient singularities.

Let $S$ be a proper surface with quotient singularities $p_{i} \in S$. Let $p_{i} \in M_{i}$ be small conical neighborhoods. For each singularity $\left(p_{i} \in M_{i}\right)$ choose a 4 -manifold such that $\partial N_{i}=\partial M_{i}$. For instance, $N_{i}$ could be the general fiber of a smoothing of $M_{i}$. In general, there need not be an algebraic deformation of $S$ which realizes these local smoothings. However, one can always get a differentiable manifold by replacing each singular $M_{i}$ with the smooth $N_{i}$. 
It is interesting to find this way differentiable manifolds which are not algebraic. This idea, though formulated purely topologically, was used in [SS05, PSS04, FS04] to construct exotic differentiable structures on $\mathbb{C P}^{2}$ blown up at $\geq 5$ points.

It is especially interesting to work with the case when each $N_{i}$ is a rational homology ball. In the early seventies, Casson, Gordon and Conway (unpublished) found 3 such classes:

(1) $\mathbb{C}^{2} / \frac{1}{n^{2}}(1, n a-1)$ where $(n, a)=1$,

(2) $\mathbb{C}^{2} / \frac{1}{n^{2}}(1, d(n-1))$ where $d \mid n-1$ is odd, and

(3) $\mathbb{C}^{2} / \frac{1}{n^{2}}(1, d(n-1))$ where $d \mid 2 n+1$.

Recently [Lis07] proved that these are in fact all, if we also take into account two elementary observations:

(4) replacing the generator of $\mathbb{Z} / p$ by its inverse shows that $\mathbb{C}^{2} / \frac{1}{p}(1, q) \cong$ $\mathbb{C}^{2} / \frac{1}{p}\left(1, q^{\prime}\right)$ for $q q^{\prime} \equiv 1 \bmod p$, and

(5) conjugating one of the coordinates shows that $\mathbb{C}^{2} / \frac{1}{p}(1, q)$ is diffeomorphic to $\mathbb{C}^{2} / \frac{1}{p}(1, p-q)$.

Two algebraic realizations of the first series were given in (21). I do not know algebraic descriptions of the other two.

Let $S$ be a proper rational homology $\mathbb{C P}^{2}$ with quotient singularities $p_{i} \in S$ which are on the list (4.1-5). The resulting topological smoothing is then a smooth 4-manifold which is also a rational homology $\mathbb{C P}^{2}$.

In an earlier version of this note I raised the possibility that such surfaces could lead to a fake $\mathbb{C P}^{2}$, that is, a smooth 4 -manifold homeomorphic but not diffeomorphic to $\mathbb{C P}^{2}$. Moreover, I was hoping to do this with smoothings that are locally complex analytic. To this end, one needs to find rational homology $\mathbb{C P}^{2}$-s with singularities of the form $\mathbb{C}^{2} / \frac{1}{n^{2}}(1, n a-1)$.

If $S$ has such singularities and $-K_{S}$ is ample, then the local deformations of the singular points can be globalized, and $S$ is the degeneration of smooth Del Pezzo surfaces. These were studied and partially classified in [Man91, HP05]. The general type examples, however, have a tendency to be rigid (cf. [FZ94, $6.12]$ ), and they may lead to new differentiable 4-manifolds. Unfortunately, we get nothing interesting. 
Theorem 35 (P. Hacking). Let $S$ be a rational homology $\mathbb{C P}^{2}$ with singularities of the form $\mathbb{C}^{2} / \frac{1}{n_{i}^{2}}\left(1, n_{i} a_{i}-1\right)$ for some $\left(n_{i}, a_{i}\right)=1$. If $K_{S}$ is nef then $S$ is smooth.

Proof. Since $K_{S}$ is nef, we have the orbifold BMY inequality $c_{1}(S)^{2} \leq 3 e_{\text {orb }}(S)$.

Noether's formula $\chi\left(\mathcal{O}_{S}\right)=\frac{1}{12}\left(c_{1}(S)^{2}+e(S)\right)$ usually needs a correction term for each quotient singularity, but for our singularities the correction term vanishes, cf. [HP05, Prop.3.5]. Thus $c_{1}(S)^{2}=9$ and so $3 \leq e_{\text {orb }}(S)$ thus $S$ is smooth.

If we also allow the other singularities on the list (4.1-5), there are interesting examples. For instance

$$
\left(u^{2}=\left(x^{2}+y^{2}-z^{2}\right)\left(x^{2}+2 y^{2}-z^{2}\right)\right) \subset \mathbb{P}^{3}(1,1,1,2)
$$

is a degree 2 Del Pezzo surface with Picard number 2 and two singular points of type $A_{3}$, that is, $\mathbb{C}^{2} / \frac{1}{4}(1,3)$. Topological smoothing creates out of it a 4 -manifold with $b_{2}=2$ (which is probably not simply connected).

It would be interesting to start a systematic study of such examples.

\section{Examples of Rational homology projective spaCes}

In this section we investigate hypersurfaces in weighted projective spaces given by an equation

$$
H\left(a_{1}, \ldots, a_{n}\right):=\left(x_{1}^{a_{1}} x_{2}+x_{2}^{a_{2}} x_{3}+\cdots+x_{n-1}^{a_{n-1}} x_{n}+x_{n}^{a_{n}} x_{1}=0\right) \subset \mathbb{P}\left(w_{1}, \ldots, w_{n}\right) .
$$

These hypersurfaces, or rather, the corresponding cones, play a fundamental role in the classification of quasi-homogeneous singularities [OR77, Kou76], but they have many other remarkable properties as well.

The best known examples arise when $a_{1}=\cdots=a_{n}=a$, giving hypersurfaces in ordinary projective space

$$
H(a):=\left(x_{1}^{a} x_{2}+x_{2}^{a} x_{3}+\cdots+x_{n-1}^{a} x_{n}+x_{n}^{a} x_{1}=0\right) \subset \mathbb{P}^{n-1} .
$$

These have been studied for their large group of automorphisms among others. (The case $a=n=3$ is Klein's curve of genus 3 with a simple group of order 168 as automorphisms.)

It turns out, however, that this case is completely misleading and for general $a_{1}, \ldots, a_{n}$ we get very different behavior. 
The closely related examples of [Lib77, BD94]

$$
\left(x_{1}^{a} x_{2}^{d-a}+x_{2} x_{3}^{d-1}+\cdots+x_{n-1} x_{n}^{d-1}+x_{n+1}^{d}=0\right) \subset \mathbb{P}^{n}
$$

give hypersurfaces with (non-quotient) isolated singularities which have the same integral homology as $\mathbb{C P}^{n-1}$ if $\operatorname{gcd}(a, d(d-1))=1$.

36 (Summary of the results). Let $H=H\left(a_{1}, \ldots, a_{n}\right)$ be as above. Then $H$ has only cyclic quotient singularities for $n \geq 4$. Under a mild but not very explicit restriction on $a_{1}, \ldots, a_{n}(38)$ we show that

(1) $H$ is birational to $\mathbb{C P}^{n-2}$, but

(2) if every $a_{i} \geq n$ and $n \geq 4$ then the canonical class $K_{H}$ is ample and its self intersection $\left(K_{H}^{n-2}\right)$ converges to 1 as $\min \left\{a_{i}\right\} \rightarrow \infty$.

Moreover, if $n$ is odd then

(3) $H$ is a rational homology $\mathbb{C P}^{n-2}$, and

(4) $\mathbb{P}\left(w_{1}, \ldots, w_{n}\right) \backslash H$ is a rational homology $\mathbb{C}^{n-1}$.

For $n=3$ this gives many examples of Seifert fibered rational homology spheres which are rationally $H$-cobordant to 0 , see (22).

If $n=2 m$ is even then $H_{n-2}(H, \mathbb{Q})$ has dimension 3. However, if $n=4$, then $H$ contains 2 disjoint contractible curves and after contracting them we get $H \rightarrow H^{*}$ and in (27) we show that

(6) $H^{*}$ is a rational homology $\mathbb{C P}^{n-2}$, and

(7) if every $a_{i} \geq 4$ then the canonical class $K_{H^{*}}$ is ample.

Remark 37. In singularity theory and in topology the Brieskorn-Pham singularities

$$
\left(x_{1}^{a_{1}}+x_{2}^{a_{2}}+\cdots+x_{n}^{a_{n}}=0\right) \subset \mathbb{C}^{n}
$$

are much better known. When the link of a Brieskorn-Pham singularity is a homology sphere, then the corresponding projective hypersurface is isomorphic to a weighted projective space [Bri66] and all the intricate geometry is concentrated in the corresponding Seifert bundle structure (see, for instance, [OW75]).

By contrast, for the singularities

$$
\left(x_{1}^{a_{1}} x_{2}+x_{2}^{a_{2}} x_{3}+\cdots+x_{n-1}^{a_{n-1}} x_{n}+x_{n}^{a_{n}} x_{1}=0\right) \subset \mathbb{C}^{n}
$$


the projective hypersurfaces are also very interesting and the Seifert bundle structure is usually simple.

38 (Numerical conditions). The exponents $a_{1}, \ldots, a_{n}$, the weights $w_{1}, \ldots, w_{n}$ and the degree $d$ are related by the equations

$$
a_{i} w_{i}+w_{i+1}=d \quad \text { for } \quad i=1, \ldots, n,
$$

where we write all subscripts modulo $n$. Let us fix the exponents $a_{i}$ and assume that $\prod a_{i} \neq(-1)^{n}$. Using (40) the system can be solved explicitly. Set

$$
W_{i}:=\sum_{j=1}^{n}(-1)^{j-1} \prod_{\ell=i+j}^{i+n-1} a_{\ell} \text { and } D:=\prod_{\ell=1}^{n} a_{\ell}+(-1)^{n-1}
$$

where $D$ is the determinant of the system. It is easy to check that

$$
a_{i} W_{i}+W_{i+1}=D \quad \text { for } \quad i=1, \ldots, n .
$$

We would like to have a well formed weighted projective space, thus we have to divide the weights by their greatest common divisor

$$
w^{*}:=\operatorname{gcd}\left(W_{1}, \ldots, W_{n}\right) .
$$

Note that the equations (38.1) imply that

$$
w^{*}=\operatorname{gcd}\left(W_{i}, D\right) \forall i \quad \text { and } \quad w^{*}=\operatorname{gcd}\left(W_{i}, W_{i+1}\right) \forall i .
$$

It turns out that the cases with $w^{*}=1$ have many special properties that are not shared by the examples with $w^{*}>1$.

It is not clear to me how to determine whether $w^{*}=1$, other than actually computing it. It is, however, easy to see that $w^{*}=1$ happens frequently.

Note that we can write $W_{n}=a_{n-1} A \pm 1$ and $D=a_{n} a_{n-1} B \pm 1$ where $A, B$ depend on $a_{1}, \ldots, a_{n-2}$ only. Fix a prime $p$ and $a_{1}, \ldots, a_{n-2}$. No matter what $A, B$ are, there is at most one choice for $a_{n-1}$ modulo $p$ such that $p$ divides $W_{n}$ and then at most one choice for $a_{n}$ modulo $p$ such that $p$ divides $D$. By the Chinese remainder theorem, the conditions for different primes are independent. Thus the proportion of the $n$-tuples with $w^{*}=1$ is asymptotically at least

$$
\prod_{p}\left(1-\frac{1}{p^{2}}\right)=\frac{1}{\zeta(2)}=\frac{6}{\pi^{2}}=0.607 \ldots
$$


Note that $D=\prod a_{i} \pm 1$ is divisible by 2 only if all the $a_{i}$ are odd, and then $w_{i} \equiv n \bmod 2$. This allows us to conclude that the above 0.607 can be improved to 0.8 if $n$ is odd and to 0.75 if $n$ is even.

Set

$$
w_{i}:=\frac{1}{w^{*}} W_{i} \quad \text { and } \quad d:=\frac{1}{w^{*}} D .
$$

From (38.3) we conclude that

$$
\operatorname{gcd}\left(w_{i}, d\right)=1 \forall i \quad \text { and } \operatorname{gcd}\left(w_{i}, w_{i+1}\right)=1 \forall i .
$$

Note that $H$ can also be viewed as a general element of the linear system

$$
|H|=\left|x_{1}^{a_{1}} x_{2}, x_{2}^{a_{2}} x_{3}, \cdots, x_{n-1}^{a_{n-1}} x_{n}, x_{n}^{a_{n}} x_{1}\right| .
$$

Indeed, take any $\lambda_{i} \neq 0$ and consider

$$
H(\lambda):=\left(\lambda_{1} x_{1}^{a_{1}} x_{2}+\lambda_{2} x_{2}^{a_{2}} x_{3}+\cdots+\lambda_{n-1} x_{n-1}^{a_{n-1}} x_{n}+\lambda_{n} x_{n}^{a_{n}} x_{1}=0\right)
$$

Choose $\mu_{i}$ such that $\mu_{i}^{a_{i}} \mu_{i+1}=\lambda_{i}^{-1}$. Such a choice is possible since we can view these equations as a linear system for the $\log \mu_{i}$ whose determinant is $D \neq 0$. Thus $H$ and $H(\lambda)$ differ only by a coordinate change.

Theorem 39. Assume that $\prod a_{i} \neq(-1)^{n}$ and write all subscripts modulo $n$. Define $w_{i}, d$ and $w^{*}$ as in (38.4). Then

(1) $\mathbb{P}\left(w_{0}, \ldots, w_{n}\right)$ is a well formed weighted projective space whose singular set has dimension $\leq\lfloor n / 2\rfloor-1$.

(2) The hypersurface

$$
H\left(a_{1}, \ldots, a_{n}\right):=\left(x_{1}^{a_{1}} x_{2}+x_{2}^{a_{2}} x_{3}+\cdots+x_{n-1}^{a_{n-1}} x_{n}+x_{n}^{a_{n}} x_{1}=0\right) \subset \mathbb{P}\left(w_{1}, \ldots, w_{n}\right)
$$

is quasi-smooth.

(3) $\mathbb{P}\left(w_{1}, \ldots, w_{n}\right) \backslash H\left(a_{1}, \ldots, a_{n}\right)$ is smooth.

(4) If $w^{*}=1$ then $H$ is birational to $\mathbb{C P}^{n-2}$.

(5) $K_{H}$ is ample if $\min \left\{a_{i}\right\} \geq n$ and $\left(K_{H}^{n-2}\right) \rightarrow 1$ as $\min \left\{a_{i}\right\} \rightarrow \infty$.

(6) If $n$ is odd and $w^{*}=1$ then

$$
\begin{aligned}
& H_{*}\left(H\left(a_{1}, \ldots, a_{n}\right), \mathbb{Q}\right) \cong H_{*}\left(\mathbb{C P}^{n-2}, \mathbb{Q}\right) \quad \text { and } \\
& H_{*}\left(\mathbb{P}\left(w_{1}, \ldots, w_{n}\right) \backslash H\left(a_{1}, \ldots, a_{n}\right), \mathbb{Q}\right) \cong H_{*}\left(\mathbb{C}^{n-1}, \mathbb{Q}\right) .
\end{aligned}
$$

(7) If $n=2 m$ is even and $w^{*}=1$ then

$$
\begin{aligned}
& H_{j}\left(H\left(a_{1}, \ldots, a_{n}\right), \mathbb{Q}\right) \cong H_{j}\left(\mathbb{C P}^{n-2}, \mathbb{Q}\right) \quad \text { for } j \neq n-2, \text { and } \\
& H_{n-2}\left(H\left(a_{1}, \ldots, a_{n}\right), \mathbb{Q}\right) \cong \mathbb{Q}^{3} .
\end{aligned}
$$


The middle homology is spanned by the the complete intersection class $c_{1}\left(\mathcal{O}_{\mathbb{P}}(1)\right)^{m-1} \cap[H]$ and the two disjoint weighted linear subspaces

$$
\left(x_{1}=x_{3}=\cdots=x_{2 m-1}=0\right) \quad \text { and } \quad\left(x_{2}=x_{4}=\cdots=x_{2 m}=0\right) .
$$

Proof. The singular locus of $\mathbb{P}\left(w_{1}, \ldots, w_{n}\right)$ is a union of weighted linear subspaces $L_{I}$ where $I \subset\{1, \ldots, n\}$ is a subset such that $\operatorname{gcd}\left\{w_{i}: i \in I\right\} \neq 1$ and

$$
L_{I}:=\left\{\left(x_{1}, \ldots, x_{n}\right): x_{j}=0 \forall j \notin I\right\} .
$$

As we noted in (38.5), $I$ does not contain any pair of indices whose difference is 1. Thus $|I| \leq n / 2$ and so $\operatorname{dim} L_{I} \leq\lfloor n / 2\rfloor-1$. We also see that $L_{I} \subset H$ for every such $I$. This shows (39.1) and (39.3).

Outside $\left(\prod x_{i}=0\right)$ the hypersurface $H$ is smooth by Bertini. Assume that $H$ is not quasi-smooth at the point $\left(p_{1}, \ldots, p_{n}\right)$ and $p_{i}=0$. Then $\partial h / \partial x_{i}=0$ shows that $p_{i-1}=0$ and by repeating the argument we get that all the $p_{j}=0$. Thus $H$ is quasi-smooth, proving (39.2).

Assuming that $w^{*}=1$, we show that the linear system

$$
|H|=\left|x_{1}^{a_{1}} x_{2}, x_{2}^{a_{2}} x_{3}, \cdots, x_{n-1}^{a_{n-1}} x_{n}, x_{n}^{a_{n}} x_{1}\right|
$$

maps $\mathbb{P}\left(w_{1}, \ldots, w_{n}\right)$ birationally to $\mathbb{P}^{n-1}$ and so $H$ is mapped birationally to a hyperplane in $\mathbb{P}^{n-1}$. Note that $|H|$ restricts to a homomorphism between the tori

$$
\eta:\left(\mathbb{C}^{*}\right)_{\mathbf{x}}^{n} \rightarrow\left(\mathbb{C}^{*}\right)_{\mathbf{y}}^{n} \quad \text { given by } \quad \eta^{*} y_{i}=x_{i}^{a_{i}} x_{i+1}
$$

where $\left(y_{1}, \ldots, y_{n}\right)$ are coordinates on the target $\mathbb{P}^{n-1}$. The degree of $\eta$ is the determinant of the matrix of exponents, which we already computed to be $D=$ $\prod_{i} a_{i}+(-1)^{n-1}$.

Let us now restrict $\eta$ to the 1 -parameter subgroup $\left(\lambda^{w_{1}}, \ldots, \lambda^{w_{n}}\right)$. We get a homomorphism of degree $d$ :

$$
\eta:\left(\lambda^{w_{1}}, \ldots, \lambda^{w_{n}}\right) \rightarrow\left(\lambda^{d}, \ldots, \lambda^{d}\right) .
$$

Note that $w^{*}=1$ iff $d=D$, thus if $w^{*}=1$ then $\eta$ descends to an isomorphism

$$
\left(\mathbb{C}^{*}\right)_{\mathbf{x}}^{n} /\left(\lambda^{w_{1}}, \ldots, \lambda^{w_{n}}\right) \rightarrow\left(\mathbb{C}^{*}\right)_{\mathbf{y}}^{n} /(\lambda, \ldots, \lambda) .
$$

This is exactly the map given by $|H|$, proving (39.4). 
Although it is not needed for our purposes, one can also write down the inverse of the map given by $|H|$. First we get the formulas

$$
\begin{aligned}
x_{i}^{D} & =\prod_{j=0}^{n-1} y_{i+j}^{b_{i j}} \quad \text { where } \quad b_{i j}:=(-1)^{j+1} \prod_{\ell=i+j-1}^{i+n-1} a_{\ell} \quad \text { and } \\
x_{i}^{w_{i+1}} x_{i+1}^{-w_{i}} & =x_{i}^{D} y_{i}^{-w_{i}} .
\end{aligned}
$$

Then one can easily check that the monomials $x_{i}^{D}$ and $x_{i}^{w_{i+1}} x_{i+1}^{-w_{i}}$ generate the subring $\mathbb{C}\left(x_{1}, \ldots, x_{n}\right)^{(D)}$ of those elements whose degree is divisible by $D$. Thus we get an explicit isomorphism

$$
|H|^{*}: \mathbb{C}\left(y_{1}, \ldots, y_{n}\right) \cong \mathbb{C}\left(x_{1}, \ldots, x_{n}\right)^{(D)} .
$$

Nevertheless, I found it very difficult to compute anything based on these formulas.

For $n \geq 5$ the singular set of $\mathbb{P}\left(w_{1}, \ldots, w_{n}\right)$ has codimension $\geq 3$, thus the canonical class of $H$ is given by the adjunction formula,

$$
K_{H}=\left.\left(K_{\mathbb{P}}+H\right)\right|_{H}=\left.\mathcal{O}_{\mathbb{P}}\left(d-\sum w_{i}\right)\right|_{H} .
$$

The minor modifications needed in the few cases when $n=4$ and $\mathbb{P}\left(w_{1}, \ldots, w_{n}\right)$ has 1-dimensional singular set are discussed in (43). If $w^{*}=1$ then the two highest terms in the coefficient of $K_{H}$ are

$$
\left(\prod a_{i}\right)\left(1-\sum \frac{1}{a_{i}}\right)
$$

which is positive as soon as $\min \left\{a_{i}\right\} \geq n$. In fact it is easy to see that $d-\sum w_{i}>0$ if $\min \left\{a_{i}\right\} \geq n$. The self intersection of $K_{H}$ is computed asymptotically by

$$
\left(K_{H}^{n-2}\right)=\left(\mathcal{O}_{\mathbb{P}}\left(d-\sum w_{i}\right)^{n-2} \cdot \mathcal{O}_{\mathbb{P}}(d)\right) \sim \frac{d^{n-1}}{\prod w_{i}} \sim w^{*} .
$$

We compute the homology groups of $H$ using the Milnor-Orlik formula [MO70].

Let $f\left(x_{1}, \ldots, x_{n}\right)$ be a weighted homogeneous polynomial of weighted degree $d$ where the variable $x_{i}$ has weight $w_{i}$. Assume that $(f=0)$ has an isolated singularity at the origin and let $L=L(f):=(f=0) \cap S^{2 n-1}(1)$ be its link. Then $L$ is $(n-3)$-connected and the rank of the middle homology groups is given by

$$
\operatorname{dim} H_{n-2}(L, \mathbb{Q})=\sum_{I \subset\{1, \ldots, n\}}(-1)^{n-|I|} \frac{\prod_{i \in I}\left(d / w_{i}\right)}{\operatorname{lcm}\left\{u_{i}: i \in I\right\}},
$$

where we write $\frac{d}{w_{i}}=\frac{u_{i}}{v_{i}}$ in lowest terms. 
In our case $\left(d, w_{i}\right)=1$ for every $i(38.5)$ an so $u_{i}=d$. Thus $\operatorname{lcm}\left\{u_{i}: i \in I\right\}=d$ save for $I=\emptyset$. Thus the formula becomes

$$
\begin{aligned}
\sum_{I \subset\{1, \ldots, n\}}(-1)^{n-|I|} & \mid \prod_{i \in I}\left(d / w_{i}\right) \\
& =(-1)^{n}+\frac{(-1)^{n-1}}{d}+\frac{1}{d} \sum_{I \subset\{1, \ldots, n\}}(-1)^{n-|I|} \prod_{i \in I} \frac{d}{w_{i}} \\
& =(-1)^{n}+\frac{(-1)^{n-1}}{d}+\frac{1}{d} \prod_{i}\left(\frac{d}{w_{i}}-1\right) \\
& =(-1)^{n}+\frac{(-1)^{n-1}}{d}+\frac{1}{d} \prod_{i}\left(\frac{a_{i-1} w_{i-1}}{w_{i}}\right) \\
& =(-1)^{n}+\frac{(-1)^{n-1}}{d}+\frac{1}{d} \prod_{i} a_{i} \\
& =(-1)^{n}+w^{*},
\end{aligned}
$$

where at the last step we took into account that $d w^{*}=D=\prod_{i} a_{i}+(-1)^{n-1}$.

The link $L$ is a Seifert $S^{1}$-bundle over $X:=(f=0) \subset \mathbb{P}\left(w_{1}, \ldots, w_{n}\right)$ and the resulting Leray spectral sequence is easy to compute (with rational coefficients), see, e.g. [OW75]. This gives (39.6-7) except for the precise identification of $H_{n-2}(H, \mathbb{Q})$ in the $n=2 m$ case.

We use this only for $n=4$, where it is worked out in (43).

Lemma 40. Assume that $\prod a_{i} \neq(-1)^{n}$ and write all subscripts modulo $n$. Then the system

$$
a_{i} v_{i}+v_{i+1}=1 \quad i=1, \ldots, n
$$

has determinant $\prod_{\ell=1}^{n} a_{\ell}+(-1)^{n-1}$ and a unique solution given by

$$
v_{i}=\frac{W_{i}}{D}:=\frac{\sum_{j=1}^{n}(-1)^{j-1} \prod_{\ell=i+j}^{i+n-1} a_{\ell}}{\prod_{\ell=1}^{n} a_{\ell}+(-1)^{n-1}} .
$$

Next we consider in greater detail the two low dimensional cases.

41 (Quasi-smooth rational curves). The case $n=3$ gives quasi-smooth rational curves in weighted projective planes. Here we have a system

$$
a_{1} w_{1}+w_{2}=a_{2} w_{2}+w_{3}=a_{3} w_{3}+w_{1}=d
$$

with solutions

$$
w_{1}=\frac{a_{2} a_{3}-a_{3}+1}{w^{*}}, w_{2}=\frac{a_{3} a_{1}-a_{1}+1}{w^{*}}, w_{3}=\frac{a_{1} a_{2}-a_{2}+1}{w^{*}}, d=\frac{a_{1} a_{2} a_{3}+1}{w^{*}} .
$$

We can also compute the genus of the general member of the linear system

$$
C \in\left|x_{1}^{a_{1}} x_{2}, x_{2}^{a_{2}} x_{3}, x_{3}^{a_{3}} x_{1}\right|
$$


using the adjunction formula (42.4). By explicit computation

$$
\frac{d\left(d-w_{1}-w_{2}-w_{3}\right)}{w_{1} w_{2} w_{3}}=w^{*}-\frac{1}{w_{1}}-\frac{1}{w_{2}}-\frac{1}{w_{3}}
$$

Thus by (42.4), the genus of $C$ is $\left(w^{*}-1\right) / 2$. Thus $C$ is a smooth rational curve iff $w^{*}=1$, that is, when $a_{2} a_{3}-a_{3}+1, a_{3} a_{1}-a_{1}+1$ and $a_{1} a_{2}-a_{2}+1$ are relatively prime.

The Kodaira dimension of the pair $\left(P:=\mathbb{P}\left(w_{1}, w_{2}, w_{3}\right), C\right)$ is determined by the sign of

$$
\begin{aligned}
\operatorname{deg}\left(C+K_{P}\right) & =a_{1} a_{2} a_{3}+1-\left(a_{1} a_{2}-a_{2}+1+a_{2} a_{3}-a_{3}+1+a_{3} a_{1}-a_{1}+1\right) \\
& =\left(a_{1}-1\right)\left(a_{2}-1\right)\left(a_{3}-1\right)-1 .
\end{aligned}
$$

This is negative if one of $a_{1}, a_{2}, a_{3}$ is 1 . If say $a_{3}=1$ then we get weighted projective planes $\mathbb{P}^{2}\left(a_{2}, 1, a_{1} a_{2}-a_{2}+1\right)$ with only 2 singular points and the corresponding link is a lens space.

If $a_{1}=a_{2}=a_{3}=2$ then the relatively prime conditions is not satisfied. In all other cases $\left(a_{1}-1\right)\left(a_{2}-1\right)\left(a_{3}-1\right)-1>0$ so the Kodaira dimension is 2 .

As a side remark we note that (22) lists all interesting quasi-smooth rational curves in weighted projective planes.

Proposition 42. Let $P:=\mathbb{P}(u, v, w)$ be a well formed weighted projective plane and $C=C_{d} \subset P$ a quasi-smooth rational curve of degree $d$. Then, up to permuting the coordinates and isomorphism, the pair $d, C$ is one of the following

(1) $d=w$ and $C=(z=0)$.

(2) $d=u+v, w \mid u+v$ and $C \in\left|x y, z^{(u+v) / w}, \ldots\right|$ where the existence of other degree $d$ monomials depends on further numerical coincidences.

(3) $(P, C)$ is as in (22).

Proof. Let $C \subset S$ be a quasi-smooth curve on a surface $S$ which passes through the singular points $P_{i}$ which are cyclic quotients by $\mathbb{Z} / m_{i}$. The adjunction formula (cf. [Cor92]) says that

$$
C\left(C+K_{S}\right)=2 g(C)-2+\sum\left(1-\frac{1}{m_{i}}\right) .
$$

Assume now that $S=P$ is a weighted projective plane, $C$ is rational of degree $d$ and it passes through at most 2 singular points of indices $u, v$, where $u=1$ or 
$v=1$ are allowed. Then we get that

$$
\frac{d(d-u-v-w)}{u v w}=-\frac{1}{u}-\frac{1}{v} .
$$

Thus $d<u+v+w$ and so if $z$ is the coordinate with the biggest weight then it appears in one of the monomials $z, z^{2}, z y, z x$. The rest follows by an easy case analysis, giving the first two possibilities.

It remains to consider the case when $C$ passes through all 3 singular points and $u, v, w \geq 2$. This gives the equation

$$
\frac{d(d-u-v-w)}{u v w}=1-\frac{1}{u}-\frac{1}{v}-\frac{1}{w} .
$$

Aside from the case $\{u, v, w\}=\{2,3,5\}$, the right hand side is positive and there is no easy upper bound for $d$.

The quasi-smoothness conditions show that, up to permuting the coordinates, we have monomials

$$
x^{a} y, y^{b} z, z^{c} x \quad \text { or } \quad x^{a} y, y^{b} x, z^{c} x .
$$

The first of these leads to (22) and to our last possibility. (In fact one can check that in the 3 singular point case, there are no other monomials with the same weighted degree.)

Finally we exclude the case $x^{a} y, y^{b} x, z^{c} x$. All of these are divisible by $x$, thus we also must have another monomial $z^{c^{\prime}} y$. Thus $\left(c-c^{\prime}\right) w=v-u$ and so $w \mid u-v$. Up to interchanging $x, y$ we can assume that $u \geq w+1$.

From $d=u a+v=b v+u$ we obtain that $(a-1) u=(b-1) v$. Since $(u, v)=1$ we get that $d=m u v+u+v$ for some $m \geq 1$. Substituting into the genus formula and rearranging we get $(m u+1)(m v+1)=(m+1) w$. But $m u+1 \geq u+1>w$ and $m v+1 \geq 2 m+1$ give a contradiction.

43 (Examples of rational homology $\mathbb{C P}^{2}$-s). For $n=4$ we get a surface

$S=S\left(a_{1}, a_{2}, a_{3}, a_{4}\right):=\left(x_{1}^{a_{1}} x_{2}+x_{2}^{a_{2}} x_{3}+x_{3}^{a_{3}} x_{4}+x_{4}^{a_{4}} x_{1}=0\right) \subset \mathbb{P}\left(w_{1}, w_{2}, w_{3}, w_{4}\right)$,

where the $a_{i}$ and $w_{i}$ satisfy a system of equations

$$
a_{1} w_{1}+w_{2}=a_{2} w_{2}+w_{3}=a_{3} w_{3}+w_{4}=a_{4} w_{4}+w_{1}=d
$$

with solutions

$$
w_{1}=\frac{a_{2} a_{3} a_{4}-a_{3} a_{4}+a_{4}-1}{w^{*}}, \ldots \quad \text { and } \quad d=\frac{a_{1} a_{2} a_{3} a_{4}-1}{w^{*}} .
$$


If $w^{*}=1$ then $S\left(a_{1}, a_{2}, a_{3}, a_{4}\right)$ is a rational surface with 4 quotient singularities at the coordinate vertices and with $H_{2}(S, \mathbb{Q}) \cong \mathbb{Q}^{3}$.

Note that $S$ contains the two rational curves

$$
C_{1}:=\left(x_{1}=x_{3}=0\right) \quad \text { and } \quad C_{2}:=\left(x_{2}=x_{4}=0\right) .
$$

Both of these are quasi-smooth in $S$. Thus by the adjunction formula (42.4),

$$
\left(K_{S}+C_{1}\right) \cdot C_{1}=-\frac{1}{w_{2}}-\frac{1}{w_{4}} \quad \text { and } \quad\left(K_{S}+C_{2}\right) \cdot C_{2}=-\frac{1}{w_{1}}-\frac{1}{w_{3}} .
$$

This implies that both curves have negative intersection with $K_{S}+(1-\epsilon)\left(C_{1}+C_{2}\right)$ for $0<\epsilon \ll 1$, and so they are are extremal rays for the $K_{S}+(1-\epsilon)\left(C_{1}+C_{2}\right)$ minimal model program. (See [KM98] for an introduction.) Thus $C_{1}$ and $C_{2}$ are both contractible to quotient singularities and we get rational surfaces

$$
\pi: S \rightarrow S^{*}=S^{*}\left(a_{1}, a_{2}, a_{3}, a_{4}\right)
$$

If the $\left\{w_{i}\right\}$ are pairwise relatively prime, then the canonical class of $S$ is

$$
K_{S}=\mathcal{O}_{\mathbb{P}}\left(\prod a_{i}-1-\sum w_{i}\right) .
$$

If the pairwise relatively prime assumption fails then the general adjunction formula [Cor92] says that

$$
K_{S}+\left(1-\frac{1}{\operatorname{gcd}\left(w_{1}, w_{3}\right)}\right) C_{1}+\left(1-\frac{1}{\operatorname{gcd}\left(w_{2}, w_{4}\right)}\right) C_{2}=\mathcal{O}_{\mathbb{P}}\left(\prod a_{i}-1-\sum w_{i}\right)
$$

Note that if $a_{1}, a_{2}, a_{3}, a_{4} \geq 4$ then $K_{S^{*}}$ is ample. One can write down an explicit formula for the self intersection of $K_{S^{*}}$, but it is rather complicated. In any case, one sees that it also converges to 1 as $a_{1}, a_{2}, a_{3}, a_{4} \rightarrow \infty$.

Acknowledgments . I thank V. Alexeev, D. Auroux, J. Amoros, W. Chen, I. Dolgachev, C. Gordon, J. Keum, T. Mrowka, A. Némethi and D.-Q. Zhang for useful comments and especially M. Zaidenberg who called my attention to numerous related papers. I am grateful to P. Hacking and J. Wahl for allowing me to use their unpublished results. J.M. Johnson helped with computer experiments that lead to the discovery of various properties of the examples in Section 5 . Partial financial support was provided by the NSF under grant number DMS0500198. 


\section{REFERENCES}

[AK79] Selman Akbulut and Robion Kirby, Mazur manifolds, Michigan Math. J. 26 (1979), no. 3, 259-284. MR MR544597 (80h:57004)

[Art75] M. Artin, Wildly ramified $Z / 2$ actions in dimension two, Proc. Amer. Math. Soc. 52 (1975), 60-64. MR MR0374136 (51 \#10336)

[Bar65] D. Barden, Simply connected five-manifolds, Ann. of Math. (2) 82 (1965), 365-385. MR MR0184241 (32 \#1714)

[BD94] Gottfried Barthel and Alexandru Dimca, On complex projective hypersurfaces which are homology- $\mathbf{P}^{n}$ 's, Singularities (Lille, 1991), London Math. Soc. Lecture Note Ser., vol. 201, Cambridge Univ. Press, Cambridge, 1994, pp. 1-27. MR MR1295070 (95j:14060)

[BGK05] Charles P. Boyer, Krzysztof Galicki, and János Kollár, Einstein metrics on spheres, Annals of Mathematics. to appear 162 (2005), no. 1, 1-24.

[Bog78] F. A. Bogomolov, Holomorphic tensors and vector bundles on projective manifolds, Izv. Akad. Nauk SSSR Ser. Mat. 42 (1978), no. 6, 1227-1287, 1439. MR MR522939 (80j:14014)

[Bri66] Egbert Brieskorn, Beispiele zur Differentialtopologie von Singularitäten, Invent. Math. 2 (1966), 1-14. MR 34 \#6788

[Bri68] _ Rationale Singularitäten komplexer Flächen, Invent. Math. 4 (1967/1968), 336-358. MR 36 \#5136

[CH81] Andrew J. Casson and John L. Harer, Some homology lens spaces which bound rational homology balls, Pacific J. Math. 96 (1981), no. 1, 23-36. MR MR634760 (83h:57013)

[Cor92] Alessio Corti, Adjunction of log divisors, Flips and abundance for algebraic threefolds (János Kollár, ed.), Société Mathématique de France, 1992, Papers from the Second Summer Seminar on Algebraic Geometry held at the University of Utah, Salt Lake City, Utah, August 1991, Astérisque No. 211 (1992), pp. 171-182. MR 94f:14013

[Dem88] Michel Demazure, Anneaux gradués normaux, Introduction à la théorie des singularités, II, Travaux en Cours, vol. 37, Hermann, Paris, 1988, pp. 35-68. MR 91k:14004

[Dol75] I. V. Dolgachev, Automorphic forms, and quasihomogeneous singularities, Funkcional. Anal. i Priložen. 9 (1975), no. 2, 67-68. MR 58 \#27958

[DR01a] Daniel Daigle and Peter Russell, Affine rulings of normal rational surfaces, Osaka J. Math. 38 (2001), no. 1, 37-100. MR MR1824902 (2002g:14096)

[DR01b] _ On weighted projective planes and their affine rulings, Osaka J. Math. 38 (2001), no. 1, 101-150. MR MR1824903 (2002h:14062)

[DR04] - On log $\mathbb{Q}$-homology planes and weighted projective planes, Canad. J. Math. 56 (2004), no. 6, 1145-1189. MR MR2102629 (2005i:14077)

[Fre82] Michael Hartley Freedman, The topology of four-dimensional manifolds, J. Differential Geom. 17 (1982), no. 3, 357-453. MR MR679066 (84b:57006)

[FS85] Ronald Fintushel and Ronald J. Stern, Pseudofree orbifolds, Ann. of Math. (2) 122 (1985), no. 2, 335-364. MR MR808222 (87a:57027) 
[FS87] _ $\mathrm{O}(2)$ actions on the 5-sphere, Invent. Math. 87 (1987), no. 3, 457-476. MR MR874031 (88e:57032)

[FS04] Ronald Fintushel and Ronald J. Stern, Double node neighborhoods and families of simply connected 4-manifolds with $b^{+}=1$, arXiv:math.GT/0412126, 2004.

[Fuj82] Takao Fujita, On the topology of noncomplete algebraic surfaces, J. Fac. Sci. Univ. Tokyo Sect. IA Math. 29 (1982), no. 3, 503-566. MR MR687591 (84g:14035)

[Fur01] M. Furuta, Monopole equation and the $\frac{11}{8}$-conjecture, Math. Res. Lett. 8 (2001), no. 3, 279-291. MR MR1839478 (2003e:57042)

[FZ94] Hubert Flenner and Mikhail Zaidenberg, Q-acyclic surfaces and their deformations, Classification of algebraic varieties (L'Aquila, 1992), Contemp. Math., vol. 162, Amer. Math. Soc., Providence, RI, 1994, pp. 143-208. MR MR1272698 (95b:14024)

[FZ03] , Normal affine surfaces with $\mathbb{C}^{*}$-actions, Osaka J. Math. 40 (2003), no. 4, 981-1009. MR 2020670

[GP99] R. V. Gurjar and C. R. Pradeep, Q-homology planes are rational. III, Osaka J. Math. 36 (1999), no. 2, 259-335. MR MR1736480 (2000m:14070)

[GS89a] R. V. Gurjar and A. R. Shastri, On the rationality of complex homology 2-cells. I, J. Math. Soc. Japan 41 (1989), no. 1, 37-56. MR MR972163 (89k:14035)

[GS89b] _ On the rationality of complex homology 2-cells. II, J. Math. Soc. Japan 41 (1989), no. 2, 175-212. MR MR984747 (90b:14045)

[HP05] Paul Hacking and Yuri Prokhorov, Degenerations of del Pezzo surfaces I, arXiv:math.AG/0509529, 2005.

[Keu07] JongHae Keum, A rationality criterion for projective surfaces-partial solution to Kollár's conjecture, Algebraic geometry, Contemp. Math., vol. 422, Amer. Math. Soc., Providence, RI, 2007, pp. 75-87. MR MR2296433

[KM98] János Kollár and Shigefumi Mori, Birational geometry of algebraic varieties, Cambridge Tracts in Mathematics, vol. 134, Cambridge University Press, Cambridge, 1998. MR MR1658959 (2000b:14018)

[KM99] Seán Keel and James McKernan, Rational curves on quasi-projective surfaces, Mem. Amer. Math. Soc. 140 (1999), no. 669, viii+153. MR MR1610249 (99m:14068)

[KNS89] Ryoichi Kobayashi, Shu Nakamura, and Fumio Sakai, A numerical characterization of ball quotients for normal surfaces with branch loci, Proc. Japan Acad. Ser. A Math. Sci. 65 (1989), no. 7, 238-241. MR MR1030189 (90k:32034)

[Kol05] János Kollár, Einstein metrics on 5-dimensional Seifert bundles, Jour. Geom. Anal. 15 (2005), no. 3, 463-495.

[Kol06] Circle actions on simply connected 5-manifolds, Topology 45 (2006), no. 3, 643-671. MR MR2218760 (2006m:57044)

[Kou76] A. G. Kouchnirenko, Polyèdres de Newton et nombres de Milnor, Invent. Math. 32 (1976), no. 1, 1-31. MR MR0419433 (54 \#7454)

[KSB88] J. Kollár and N. I. Shepherd-Barron, Threefolds and deformations of surface singularities, Invent. Math. 91 (1988), no. 2, 299-338. MR MR922803 (88m:14022) 
[Lib77] A. S. Libgober, A geometrical procedure for killing the middle dimensional homology groups of algebraic hypersurfaces, Proc. Amer. Math. Soc. 63 (1977), no. 2, 198-202. MR MR0440576 (55 \#13450)

[Lis07] Paolo Lisca, Lens spaces, rational balls and the ribbon conjecture, Geom. Topol. 11 (2007), 429-472. MR MR2302495

[Man91] Marco Manetti, Normal degenerations of the complex projective plane, J. Reine Angew. Math. 419 (1991), 89-118. MR MR1116920 (92f:14028)

[Mat82] Yukio Matsumoto, On the bounding genus of homology 3-spheres, J. Fac. Sci. Univ. Tokyo Sect. IA Math. 29 (1982), no. 2, 287-318. MR MR672065 (84g:57010)

[Meg92] Gábor Megyesi, Chern classes of Q-sheaves, Flips and abundance for algebraic threefolds (János Kollár, ed.), Société Mathématique de France, 1992, Papers from the Second Summer Seminar on Algebraic Geometry held at the University of Utah, Salt Lake City, Utah, August 1991, Astérisque No. 211 (1992), pp. 115-126. MR 94f:14013

[Miy77] Yoichi Miyaoka, On the Chern numbers of surfaces of general type, Invent. Math. 42 (1977), 225-237. MR MR0460343 (57 \#337)

[Miy84] The maximal number of quotient singularities on surfaces with given numerical invariants, Math. Ann. 268 (1984), no. 2, 159-171. MR MR744605 (85j:14060)

[Miy01] Masayoshi Miyanishi, Open algebraic surfaces, CRM Monograph Series, vol. 12, American Mathematical Society, Providence, RI, 2001. MR 2002e:14101

[MO70] John Milnor and Peter Orlik, Isolated singularities defined by weighted homogeneous polynomials, Topology 9 (1970), 385-393. MR MR0293680 (45 \#2757)

[MT87] M. Miyanishi and S. Tsunoda, Open algebraic surfaces with Kodaira dimension $-\infty$, Algebraic geometry, Bowdoin, 1985 (Brunswick, Maine, 1985), Proc. Sympos. Pure Math., vol. 46, Amer. Math. Soc., Providence, RI, 1987, pp. 435-450. MR MR927966 (89d:14051)

[MY72] Deane Montgomery and C. T. Yang, Differentiable pseudo-free circle actions on homotopy seven spheres, Proceedings of the Second Conference on Compact Transformation Groups (Univ. Massachusetts, Amherst, Mass., 1971), Part I (Berlin), Springer, 1972, pp. 41-101. Lecture Notes in Math., Vol. 298. MR MR0362383 (50 \#14825)

[Neu80] Walter D. Neumann, An invariant of plumbed homology spheres, Topology Symposium, Siegen 1979 (Proc. Sympos., Univ. Siegen, Siegen, 1979), Lecture Notes in Math., vol. 788, Springer, Berlin, 1980, pp. 125-144. MR MR585657 (82j:57033)

[NN02] András Némethi and Liviu I. Nicolaescu, Seiberg-Witten invariants and surface singularities, Geom. Topol. 6 (2002), 269-328 (electronic). MR MR1914570 (2003i:14048)

[NN04]_, Seiberg-Witten invariants and surface singularities. II. Singularities with good $\mathbb{C}^{*}$-action, J. London Math. Soc. (2) 69 (2004), no. 3, 593-607. MR MR2050035 (2005g:14070)

[NN05] _ Seiberg-Witten invariants and surface singularities: splicings and cyclic covers, Selecta Math. (N.S.) 11 (2005), no. 3-4, 399-451. MR MR2215260

[NW90] Walter Neumann and Jonathan Wahl, Casson invariant of links of singularities, Comment. Math. Helv. 65 (1990), no. 1, 58-78. MR MR1036128 (91c:57022) 
[OR77] P. Orlik and R. Randell, The structure of weighted homogeneous polynomials, Several complex variables (Proc. Sympos. Pure Math., Vol. XXX, Part 1, Williams Coll., Williamstown, Mass., 1975), Amer. Math. Soc., Providence, R. I., 1977, pp. 57-64. MR MR0447229 (56 \#5544)

[Ore97] S. Yu. Orevkov, Acyclic algebraic surfaces bounded by Seifert spheres, Osaka J. Math. 34 (1997), no. 2, 457-480. MR MR1483860 (99b:14033)

[OW75] Peter Orlik and Philip Wagreich, Seifert n-manifolds, Invent. Math. 28 (1975), 137159. MR MR0361150 (50 \#13596)

[OZ99] Keiji Oguiso and De-Qi Zhang, On the complete classification of extremal log Enriques surfaces, Math. Z. 231 (1999), no. 1, 23-50. MR MR1696755 (2000e:14059)

[Pet75] Ted Petrie, Equivariant quasi-equivalence, transversality and normal cobordism, Proceedings of the International Congress of Mathematicians (Vancouver, B. C., 1974), Vol. 1, Canad. Math. Congress, Montreal, Que., 1975, pp. 537-541. MR MR0431234 (55 \#4235)

[Pin77] H. Pinkham, Normal surface singularities with $C^{*}$ action, Math. Ann. 227 (1977), no. 2, 183-193. MR 55 \#5623

[PSS05] Jongil Park, András I. Stipsicz, and Zoltán Szabó, Exotic smooth structures on $\mathbb{C P}^{2} \# 5 \overline{\mathbb{C P}^{2}}$, Math. Res. Lett. 12 (2005), no. 5-6, 701-712. MR MR2189231 (2006i:57059)

[PY07] Gopal Prasad and Sai-Kee Yeung, Fake projective planes, Invent. Math. 168 (2007), no. 2, 321-370. MR MR2289867

[Rei78] Miles Reid, Bogomolov's theorem $c_{1}^{2} \leq 4 c_{2}$, Proceedings of the International Symposium on Algebraic Geometry (Kyoto Univ., Kyoto, 1977) (Tokyo), Kinokuniya Book Store, 1978, pp. 623-642. MR MR578877 (82b:14014)

[Sak80] Fumio Sakai, Semistable curves on algebraic surfaces and logarithmic pluricanonical maps, Math. Ann. 254 (1980), no. 2, 89-120. MR MR597076 (82f:14031)

[Sav02] Nikolai Saveliev, Invariants for homology 3-spheres, Encyclopaedia of Mathematical Sciences, vol. 140, Springer-Verlag, Berlin, 2002. MR MR1941324 (2004c:57026)

[Sco83] Peter Scott, The geometries of 3-manifolds, Bull. London Math. Soc. 15 (1983), no. 5, 401-487. MR MR705527 (84m:57009)

[Sei32] Herbert Seifert, Topologie dreidimensionaler gefaserte Räume, Acta Math. 60 (1932), 148-238.

[Sho00] V. V. Shokurov, Complements on surfaces, J. Math. Sci. (New York) 102 (2000), no. 2, 3876-3932. MR 2002c:14030

[Sma62] Stephen Smale, On the structure of 5-manifolds, Ann. of Math. (2) 75 (1962), 38-46. MR 25 \#4544

[SS05] András I. Stipsicz and Zoltán Szabó, An exotic smooth structure on $\mathbb{C P}^{2} \# 6 \overline{\mathbb{C P}}^{2}$, Geom. Topol. 9 (2005), 813-832 (electronic). MR MR2140993

[SSW06] András I Stipsicz, Zoltán Szabó, and Jonathan Wahl, Rational blow-downs and smoothings of surface singularities, arXiv:math.GT/0611157, 2006. 
[ST80] Herbert Seifert and William Threlfall, Seifert and Threlfall: a textbook of topology, Pure and Applied Mathematics, vol. 89, Academic Press Inc. [Harcourt Brace Jovanovich Publishers], New York, 1980. MR 82b:55001

[Ste78] Ronald J. Stern, Some more Brieskorn spheres which bound contractible manifolds, Notices Amer. Math. Soc. 25 (1978), A448.

[tDP89] Tammo tom Dieck and Ted Petrie, Homology planes: an announcement and survey, Topological methods in algebraic transformation groups (New Brunswick, NJ, 1988), Progr. Math., vol. 80, Birkhäuser Boston, Boston, MA, 1989, pp. 27-48. MR MR1040856 (91d:14016)

[Wah80] Jonathan Wahl, Elliptic deformations of minimally elliptic singularities, Math. Ann. 253 (1980), no. 3, 241-262. MR MR597833 (82d:14004)

[Wah81] S Smoothings of normal surface singularities, Topology 20 (1981), no. 3, 219246. MR MR608599 (83h:14029)

[Wah83] _ letter to W. Neumann, unpublished, 1983.

[Wah90] A A characteristic number for links of surface singularities, J. Amer. Math. Soc. 3 (1990), no. 3, 625-637. MR MR1044058 (91c:14043)

[Wah94] _ Miyaoka-Yau inequality for normal surfaces and local analogues, Classification of algebraic varieties (L'Aquila, 1992), Contemp. Math., vol. 162, Amer. Math. Soc., Providence, RI, 1994, pp. 381-402. MR MR1272710 (95b:14025)

[Wol67] Joseph A. Wolf, Spaces of constant curvature, McGraw-Hill Book Co., New York, 1967. MR MR0217740 (36 \#829)

[Yau77] Shing Tung Yau, Calabi's conjecture and some new results in algebraic geometry, Proc. Nat. Acad. Sci. U.S.A. 74 (1977), no. 5, 1798-1799. MR MR0451180 (56 \#9467)

János Kollár

Department of Mathematics, Fine Hall

Washington Road, Princeton University, Princeton NJ 08544-1000, USA

E-mail: kollar@math.princeton.edu 